\title{
Research on the Development Mode of Sports Industry in Gannan Prefecture
}

\author{
Shangjun Liu, Jiyuan Tang \\ Department of Physical Education, Gansu Normal University for Nationalities, Hezuo Gansu \\ 747000, China
}

Keywords: Gannan Prefecture sports industry development model

Abstract: With the rapid development of China's economy, the sports industry has also developed rapidly. It has gradually become a new growth point in the development of China's national economy, and it has broad development prospects. In order to promote the rapid and effective development of the sports industry in Ganzhou South, this paper comprehensively uses research methods such as literature research and investigation to conduct an in-depth study on the development model of sports industry in Gannan. This paper analyzes the current situation of Gannan's sports industry development model, points out its existing problems, and points out the sports industry model that Gannan Prefecture will develop.

\section{Research objects and research methods}

\subsection{Research object}

Taking the development model of sports industry in Gannan as the object of study

\subsection{Research methods}

\subsubsection{Literature data method}

Check related documents and materials through the Internet, libraries, etc., collect, sort, and classify a large amount, do this research and thesis report, and provide theoretical basis for the research.

\subsubsection{Questionnaire survey method}

According to the research purpose of this topic, according to the theories and methods of relevant disciplines, various indicators are set up in a targeted manner. The establishment of the indicators requires the consultation of relevant experts and scholars, and can be established after repeated selection and modification. Questionnaire issuance and recovery, will be used on-site distribution and recovery questionnaire. 120 questionnaires were distributed, 117 were recovered, 115 valid questionnaires, with an efficiency of $98.2 \%$.

\subsubsection{Interview}

On November 7-9, 2017, I visited the Gannan State Sports Bureau and the sports bureaus of various counties to learn about the development model of the sports industry in Gannan. 


\section{Research content}

\subsection{Sports Industry}

The sports industry known as the "Chaoyang Industry" is one of the hottest industries in various countries in the world today. The so-called sports industry refers to the entirety of the same type of economic activities that provide sports products to society and the integration of similar economic sectors. Sports products in the sports industry include two parts, namely tangible sports goods and intangible sports services. The sports economic department includes various other institutions engaged in business activities by market enterprises, such as sports industry institutions or social groups and individuals in the sports industry.

\subsection{Development model of sports industry}

The development mode of sports industry refers to the specific industrial structure formed by the development mode of sports industry among regions, the form of sports industry resources and the dynamic contact mechanism between the evolution path of time sequence in the region and sports industry departments. For this reason, the development mode of regional sports, the comprehensive choice of regional sports industry resources, the time sequence of regional development and the choice of regional leading industry of sports industry can be regarded as the basic components of sports development mode. The development of sports industry in a certain region or region is a dynamic process of flow and opening, and its capital also has certain liquidity.

\subsection{Current status of Gannan sports industry development model}

Gannan Prefecture not only has rich and superior natural tourism resources, but also has unique regional cultural and cultural resources. According to the survey, 45\% of people think that Gannan has a sports industry, 33\% think that Gannan has fewer sports industries, and $22 \%$ think that Gannan has no sports industry. As far as the current development of Gannan Prefecture is concerned, there are some imbalances in economic and social development. Gannan Prefecture adopts an unbalanced development method to promote the effective development of the sports industry in Gannan Prefecture. Gannan Prefecture can achieve the development of Gannan Prefecture by focusing on the actual situation of its own development, while fully considering the objective development differences, and giving priority to the capital, technology and related resource conditions in the economic center of Gannan sports industry development, thereby driving various the rapid improvement of the overall level of the industry. In addition, Gannan Prefecture can also effectively explore the choice of the development mode of the sports industry in Gannan Prefecture by combining various local economic, social, cultural, specific environment, sports resources and other conditions. According to 2018 statistics, Gannan Prefecture has 26 cultural industries, 83 economic industries, and 4 sports industries.

\subsection{Large-scale competitive sports model}

The sports model to be developed in Gannan will inevitably have large-scale competitive sports models. Because large-scale competitive sports can strongly influence and strengthen people's sports values, and promote the development of sports fitness, leisure and entertainment industries, sports tourism and other industries. In recent years, large-scale sports events held in Gannan have Hercules competitions, which are relatively large and relatively high; horse racing, which is relatively large, with more than 10,000 participants; the first national plateau mountain crossing challenge, which is relatively large and relatively high. 
From these projects, it can be seen that the effect of sports competitions in Gannan State is amplified, which in turn causes structural changes and growth of the original industry and promotes local economic development. Gannan Prefecture is an underdeveloped region in China, with relatively low per capita disposable income. However, the use of large-scale competitive sports as the core industry development model in Gannan Prefecture can effectively play an active role, which can have a greater pulling effect on the development of Gannan's sports industry, thereby improving the local economic level and increasing people’s per capita income.

\subsection{Professional sports club mode}

The mode of professional sports club is a development mode that Gannan will adopt. Because the sports training service industry is an important industry in the sports demand chain, professional sports clubs can provide certain sports training service industry to meet the needs of professional sports people to a certain extent. Through this kind of intermediate demand can also stimulate the demand expansion of other industries, which makes the inter industry correlation effect of Gannan further expand.

Gannan County clubs and professional sports clubs are also developing. Gannan Prefecture has special development conditions and related resources. The professional sports club mode is also a practical and feasible development mode. Gannan Prefecture has quite high comprehensive advantages in location conditions, natural climate, stadium construction scale and facilities, brand effect, and has a greater attraction for professional sports at home and abroad. It will promote the development of sports industry and related industries in Gannan Prefecture, stimulate the development of other industries to a certain extent, and improve the local economic level and people's living standards.

\subsection{Sports leisure and fitness mode}

From the current development mode of sports industry in Gannan Prefecture, it is generally composed of noumenon industry, related industry and other industries. In Gannan sports industry, the development of sports goods manufacturing industry is relatively slow. And there are many deficiencies in design ability, product technical ability and so on. At the same time, the development mode of sports industry in Gannan Prefecture is not harmonious. In this regard, Gannan Prefecture should constantly optimize the development mode of sports industry according to the current actual development situation, and respond to the market requirements for the development of sports industry with the best and most suitable development mode of local sports industry.

Sports leisure and fitness mode will also be a new mode of sports industry development in Gannan Prefecture. In recent years, with the continuous development of local economy in Gannan Prefecture, people's income level continues to improve, and people's quality of life is also constantly improving. People's taste and pursuit in sports, entertainment and leisure are also higher and higher. More and more people participate in sports, leisure and fitness activities. Therefore, we can effectively grasp the current people's pursuit of the hot spot, to the best of it, so that it is possible to promote the leapfrog development of sports industry.

\subsection{Sports tourism mode}

In recent years, due to the rapid improvement of Gannan's economic level, the local people's economic income is also greatly increased. The local people's demand for sports and entertainment industry is growing. In this regard, it is necessary to carry out a targeted overall planning and layout for the development mode of sports industry in Gannan Prefecture, and conduct necessary research 
and evaluation on the specific market environment in advance. In this way, we can better promote the development of sports industry in Gannan Prefecture.

In addition to a large number of sports modes of competition, competition and fitness, it is also exploring and developing other sports modes. In fact, the sports mode of competition, competition and fitness can be considered as the phenomenon of repeated construction to a certain extent. Therefore, Gannan will also develop innovative sports mode. In this context, Gannan Prefecture will also develop sports tourism mode. Because Gannan Prefecture has superior geographical location, social and cultural environment, national sports resources and unique tourism resources for sightseeing and leisure, the combination of tourism resources and sports can effectively promote the development of both. At the same time, Gannan Prefecture should further develop and innovate ideas, constantly broaden the connotation of tourism, deeply and practically develop diversified tourism products, and constantly optimize the tourism industrial structure.

\section{Conclusions and recommendations}

\subsection{Conclusion}

In the development of the sports industry in Gannan Prefecture, it is necessary to fully integrate the actual development of the local area, scientifically make overall planning, integrate effective development resources, formulate and select the best sports industry development model, and seek to achieve leapfrog development. Since the current sports industry in Gannan is in its infancy, there are still many imperfections in many areas.

\subsection{Recommendation}

In this regard, we must constantly correct ourselves and improve ourselves. At the same time, it should not be limited to the development of a single field, but should be oriented to multi-fields, with technology as the core and support, using the combination of new technologies and sports flexibly and effectively, and continuing to innovate and win a broader development market. Only in this way can the national fitness be promoted, and the improvement of the national physical fitness can be promoted. At the same time, it can also promote the effective development of the sports industry, thereby improving the level of economic development.

\section{References}

[1] Zhao Wen. Study on the development mode of Shanghai Sports Industry [D]. Wuhan Institute of physical education, 2006

[2] Zhou Aiguang, Du Gaoshan. Research on the development of China's sports industry from the perspective of the new normal [J]. Journal of sports, 2016, 23 (6): 7-13

[3] Yu Liangnan, Hu Hongbin. Research on development mode of urban sports industry [J]. Theoretical research on Urban Construction: electronic edition, 2013 (28)

[4] Li Kunxian. Research on the development mode of China's sports industry from the perspective of broad economics

[J]. Career, 2013 (36): 160-161

[5] Chen Ying. Research on the development mode of traditional Qiang sports industry in Sichuan Province [J]. Science and technology economic guide, 2015 (3)

[6] Yang Tao. Research on development mode of sports industry in different regions of Shaanxi Province [J]. China Sports Science and technology, 2009, 45 (5): 94-98

[7] Ye Chenxi. Research on the integration mode of sports industry and tourism industry in Henan Province [J]. Sports culture guide, 2017 (8)

[8] Zhang Huimin. Research on o2o model in sports industry [J]. Sports culture guide, 2017 (3)

[9] Li Kunxian. Research on the development model of China's sports industry from the perspective of broad economics

[J]. Career, 2013 (36): 160-161 